\title{
ON-LINE PARALLEL PROCESSING FOR A ROTATING POSITRON TOMOGRAPH OPERATED IN 3D MODE
}

\author{
C. Comtat, M. Egger ${ }^{* *}$, A. Herrmann Scheurer ${ }^{* *}$, \\ C. Joseph, C. Morel1) \\ Institute for Nuclear Physics, University of Lausanne, Lausanne, Switzerland \\ R.W. Dobinson, F.F. de Jong*), A.J.E. van Nieuwenborg*), J.L. Pagès2), \\ M.I. Williams $\left.{ }^{4}\right)^{*}$ ) \\ European Laboratory for Particle Physics (CERN), \\ Geneva, Switzerland \\ D.W. Townsend 3$)^{* * *}$ ) \\ Division of Nuclear Medicine, University Hospital, Geneva, Switzerland
}

\begin{abstract}
The design and implementation of an on-line parallel processing system for the rotating positron tomograph RPT-1 are presented. A unique property of this scanner is the fact that complete projections become available at regular intervals during data acquisition. This was exploited in such a way as to start reconstruction before the end of data acquisition. Readout of the data from the scanner, data normalization, and image reconstruction are pipelined in separate processing stages. A transputer-based multiprocessor network and real-time operating system provide a parallel environment which can evolve and scale to match the needs of a rotating positron tomograph.
\end{abstract}

(Submitted to Nuclear Instruments and Methods in Physics Research)

\footnotetext{
1) Present address: Division of Nuclear Medecine, University Hospital, CH-1211 Geneva 4, Switzerland.

2) Present address: IBM Switzerland, av. Giuseppe-Motta 48, CH-1202 Geneva, Switzerland.

3) Present address: PET Facility, University of Pittsburgh Medical center, Pittsburgh PA 15213, USA.

4) Present address: Department of Physics, University of Liverpool, Liverpool L69 3BX, U.K.

*) Supported by the ESPRIT III OMI/Harmony project CE7253.

${ }^{* *}$ Supported by the Swiss Federal Office of Education and Sciences (OFES), Grant E3260 for the ESPRIT III OMI/Harmony project CE7253.

${ }^{* * *}$ Supported by the Swiss Commission for the Encouragement of Scientific Research (CERS), Grant 2250.1.
} 
Positron Emission Tomography (PET) is an in vivo imaging technique in which a radiopharmaceutical labelled with a positron emitting isotope is administered intravenously or by inhalation to the patient. A PET scanner is used to image the spatial distribution of this radiopharmaceutical.

A positron resulting from isotope decay annihilates with an electron in the surrounding body tissue, producing two $511 \mathrm{keV}$ photons travelling in opposite directions. The PET scanner is composed of a number of adjacent detector rings which detect these annihilation photon pairs in coincidence. The resulting information allows an image of the density of the annihilation points to be constructed approximating the spatial distribution of the tracer.

Multi-ring scanners for PET have hecome available incorporating a three-dimensional (3D) mode of operation in addition to the more conventional two-dimensional (2D) mode. In 2D mode, only photons within the planes of the rings are acquired, i.e. the two detector cells used to register the coincident arrival of the two photons lie in the same ring, and define a line of response (LOR) - the coincidence channel-in the plane of the detector ring perpendicular to the scanner axis.

In $3 \mathrm{D}$ mode, photons with trajectories oblique to the planes of the detector rings are also acquired and more efficient use is then made of the available photon flux. An oblique line of response is no longer perpendicular to the scanner axis, but makes an angle $\pi / 2-\theta$ with it. The corresponding two detector cells lie in different rings, the angle $\theta$ being related to their ring index difference (Fig. 1).

The appearance of 3D PET scanners created a demand for fast 3D volume reconstruction. However, the large amount of data to be processed remains an obstacle to the more widespread use of 3D imaging. The reconstruction of a $128 \times 128 \times 31$ voxel image from a 16-ring scanner takes about two hours on a Sun SparcStation 10. Various techniques have been employed to accelerate reconstruction. One system [1] based on an Intel i860 Supercard can reconstruct such an image in 30 minutes using the reprojection algorithm of Kinahan and Rogers [2, 3]; to achieve this performance it is necessary to code time-consuming segments in assembler. Another approach, an implementation [4] of the Fast Volume Reconstruction algorithm (FaVoR) [5] using a network of 44 Inmos T 800 transputers, takes 20 minutes and is written entirely in a high-level language.

Recently, more cost-effective clinical PET scanner designs have heen developed in which two rotating, opposed arrays of detectors are used instead of the more conventional full rings $[6,7]$. As a consequence of this particular partial ring geometry, sets of data become available for image reconstruction at regular intervals during data acquisition. Data processing can thus start before the end of data acquisition, a property unique to partial-ring scanners.

Real-time acquisition techniques and on-line parallel data processing methods employed in high-energy physics experiments [8] are of direct relevance to medical imaging applications. This work presents the design and implementation of a transputer-based on-line parallel processing system for a rotating positron tomograph. We demonstrate how the possibility to process data before the end of the acquisition is exploited, allowing all corrections, and image reconstruction to be performed on-line-in parallel with acquisition. Prospects for improving the performance of this system to achieve real-time reconstruction are also discussed. 


\section{THE 3D ROTATING POSITRON TOMOGRAPH RPT-1}

The rotating positron tomograph RPT-1 [6] was designed to explore the potential of rotating arrays and was built based on BGO block detectors [9]. Each BGO block is cut into 8 by 8 individual detector cells, and read out by four photomultiplier tubes. Light sharing between the photomultiplier tubes is used to identify the active detector cell. RPT-1 contains only one third of the number of detector cells in an equivalent full-ring scanner with 16 rings of 384 detector cells per ring, such as the positron tomograph ECAT 953B [10]. The detectors are arranged as two opposing arrays, each of 16 axial and 64 transaxial detector cells, and every detector cell is in coincidence with every detector cell in the opposing array. The detector arrays are rotated to collect data for all discrete azimuthal angles $\phi$ of the LORs. Data are acquired for a predetermined time at a preset number of equally spaced angular positions of the detector arrays. Coincidences for the same LOR may be acquired with different pairs of detector cells at different angular positions of the detector arrays.

The number of coincidences for each LOR is stored in sinograms. A sinogram is a twodimensional matrix in which each LOR has an address specified by its distance to the scanner axis $s$ and its azimuthal angle $\phi$, for a given ring index difference. Coincidences between detector cells within the same ring or in any two different rings are stored in a frame comprising $256(16 \times 16)$ separate sinograms. During data acquisition, sinograms are progressively assembled for coincidences detected at each angular position of the detector arrays.

At each angular position the pair of detector arrays operating in coincidence acquires data within a diamond-shaped region of each sinogram (Fig. 2a). As the detector arrays rotate this diamond-shaped region shifts down the angular ordinate axis of the sinograms (Fig. 2b). The actual radial extent of the useful field of view (FOV) depends on the overlap between consecutive angular positions of the detector arrays (Fig. 2c). An acquisition or scan carried out in 12 angular positions results in a FOV of $30.1 \mathrm{~cm}$, whereas fewer angular positions are needed when the required FOV is smaller.

The effect of overlapping areas of the diamond-shaped regions acquired at different angular positions of the detector arrays must be corrected for, as well as the effect of radioactive decay on the coincidence rate measured at each position. In addition to these corrections [11], data must also be corrected for detector efficiency, detector dead-time, and photon attenuation $[12,13]$.

\section{3}

\section{ON-LINE PARALLEL PROCESSING OF THE RPT-1 DATA}

In the original configuration which was the starting point for our development, with the exception of attenuation correction which takes place off-line, corrections, are carried out simultaneously with data acquisition by the controlling computer. Data are read out after each rotation of the detector arrays and corresponding intermediate sinogram data are assembled in memory. Image reconstruction does not begin before the end of data acquisition, it takes place off-line. In the on-line processing system presented here, readout, corrections and image reconstruction are pipelined in separate processing stages, and corresponding intermediate sinogram data are buffered entirely in memory [14].

While detector arrays move from one angular position to the next, data acquired for the previous angular position are read out, then corrected and assembled into sinograms. The 
overlap between sinogram data acquired at each position depends on the number of angular positions chosen to build the frame (Fig. 2c). As shown in Figure 3, once the detector arrays have rotated by $60^{\prime \prime}$ from the first angular position, no further coincidences are stored in a rectangular sinogram area which is therefore available for image reconstruction. An equal-sized rectangular sinogram area will be available for reconstruction after each rotation of the detector arrays until they reach the last angular position of the scan. If image reconstruction cannot keep up with data collection, available sinogram data must be queued in a buffer before being processed. Figure 4 shows the complete on-line processing pipeline from data acquisition to image reconstruction.

The on-line processing system was developped using transputers as building blocks. A transputer is a microprocessor with on-chip serial links and hardware support for process scheduling [15]. Transputers are easily assembled into networks appropriate for each problem. The tasks of readout, correction, buffering, and image reconstruction are implemented in the $\mathrm{C}$ language on interconnected sub networks of transputers, each performing a different function. Commercial Siemens-CTI acquisition electronics are used for coincidence processing, and the coincidence events are histogrammed in VME memory. VME memory readout is achieved via a master/slave transputer-based VME module, an IMS B016-4 with 16 Mbyte onboard memory [16].

\subsection{Pipelined data handling using CHORUS and transputers}

Two T805 transputers with 16 Mbyte external memory apply corrections to the data read out by the B016, and assemble them into sinograms. When a sinogram area is ready for image reconstruction, the corresponding sinogram rows are sent to the buffer transputers to await analysis by the reconstruction network. Data buffering is performed by 4 interconnected T805 transputers each with 16 Mbyte external memory contained within a VOLVOX-TW800 [17]. They buffer sinogram rows in memory, allowing the readout, correction, and image reconstruction processes to run asynchronously.

The CHORUS (MiXlite) modular distributed operating system is an implementation of UNIX with real-time programming extensions [18]. Running on the nodes of the VOLVOXTW800, it provides support for asynchronous inter process communication and priority scheduling, which greatly facilitates the implementation of the buffer manager. All transfers to and from the buffer transputers are handled by two T805-16Mbyte transputers.

The buffer manager performs two parallel tasks. The first task receives and stores data in memory and the second serves data requests from the reconstruction network. The processes involved in receiving and storing data operate at a high priority, whereas those that read data and forward them to the reconstruction network run at a lower priority. In this way, the processes that send data to the reconstruction network do not interfere with the processes that receive data, although they all run on the same transputers, and the first task can keep up with the incoming data flow.

\subsection{D image reconstruction}

Exact 3D image reconstruction requires the measurement of 2D parallel projections of the volume being imaged. Therefore sinogram data have to be sorted into parallel sets of LORs, thus building 2D parallel projections of the $3 \mathrm{D}$ tracer distribution. Among all the 2D parallel 
projections assembled in this way, only the projections perpendicular to the scanner axis-the direct projections-are complete measurements of $2 \mathrm{D}$ parallel projections of the 3D tracer distribution. All the others-the oblique projections-are truncated due to the cylindrical geometry of the scanner. This intrinsic difference between the direct and the oblique projections requires direct and oblique sinogram data to be treated differently.

3D image reconstruction is performed by a parallel implementation of the Fast Volume Reconstruction (FaVoR) algorithm on a network of $36 \mathrm{~T} 800$ transputers. Details of the FaVoR algorithm and its implementation using a network of transputers have been fully discussed in Ref. [4]. Minor changes to the order in which the data are processed were necessary to adapt the original implementation of $\mathrm{FaVoR}$ to the case of the rotating scanner.

As shown in Figure 5a, direct and oblique projection data are processed separately on different trees of the network. Oblique projections undergo centralized ID filtering before their contribution to the image is restored, this computationally intensive task being shared by 30 processors that are each assigned a thirtieth of the image volume (Fig. 5b). The parallelization of the processing of the direct projections is somewhat different: multiple filtering in $2 \mathrm{D}$ of the data requires most of the processing time, so that every processor is assigned a group of complete image slices, and performs both 2D filtering and the corresponding backprojection of the direct projections into these image slices. This part uses fewer processors than the oblique part since direct projection data represent only a small fraction of the frame.

\section{DISCUSSION AND PROSPECTS}

For a 12 minute scan, an end-to-end time of 25.5 minutes from the start of a scan until the image is available is achieved. This time is dominated by the reconstruction of the image, which takes about 20 minutes with the present T800 transputer network, regardless of the scan duration. As an example, Figure 6 shows timings and data volumes for a 12-position scan of 12 minutes. 512-kbyte blocks of sinogram data become ready for reconstruction at each of the eight scan positions from the fourth one onwards. At the end of the scan the remaining data are transferred as one 2-Mbyte block. After each rotational position, readout of the VME memory (4 Mbytes of data), corrections, and sinogram assembly take about 25 seconds. Once sinogram rows are ready for reconstruction, they are buffered in the VOLVOX-TW800) until they can be accepted by the reconstruction network. Although the processing of the direct projections represents only $20 \%$ of the sinogram data, it is slightly slower than the processing of the oblique projection. The feasibility of an improved farming method with better inherent load balancing is currently being investigated.

Timing tests were carried out with the new Inmos T9000) transputer. The available chips were gamma releases operating at $12.5 \mathrm{MHz}$ and $20 \mathrm{MHz}$, with 16 kbyte cache. The test program performed 2D filtering of direct projections as well as backprojection of the data on one slice of the image. By extrapolating the clock speed of the T900)( $50 \mathrm{MHz}$, computation times are reduced by a factor of 10 as compared to a T800 running at $25 \mathrm{MHz}$. As communications over the links will also be improved by a factor of 5 , they would not become a bottleneck. By applying T9000s to the on-line PET processing system a 3D reconstructed image could be viewed minutes after the end of a scan. 
A further advantage of a farmed configuration is the possibility to use the processing power of the transputer network for further online analysis of images when it is not used by the image reconstruction. This would be advantageous when frame acquisition times longer than the reconstruction time are used, in which case reconstruction utilizes only a fraction of the maximum computing power.

It has already been demonstrated that the scaling properties of transputer networks may be exploited in order to achieve comparable reconstruction times for 24- and 32-ring scanners [19]. Similar techniques could be applied to the entire on-line PET processing system to adapt it for use with such large scanners.

\section{CONCLUSION}

An evolutive and scaleable parallel system matching the constraints imposed by the rotating positron tomograph RPT-I has been presented. It is assembled using a transputerbased multiprocessor network and a real-time operating system.

An end-to-end time of 25 minutes from the start of a frame until the image is available has been achieved, for a 12 minute frame duration. The overall processing time is still dominated by the reconstruction of the image with the present T800) transputer network. Using imminently available T9000 transputers, the reconstruction would be able to keep up with the acquisition, and the image could be viewed minutes after the end of the acquisition.

\section{Acknowledgements}

We wish to thank Prof. A. Donath, Head of the Division of Nuclear Medicine of the Geneva University Hospital, who kindly provided access to the PET scanner facility. We also gratefully thank L. Byars for technical information on the acquisition software, and M. Defrise for fruitful discussions on reconstruction algorithms. 


\section{References}

[1] M.S. Atkins, M. Zastre, K. Buckley and L. Byars, Evaluation of the use of the i860) supercard in a 3D PET tomograph, in Conf. Records 1992 IEEE Medical imag. Conf., Orlando, FL, 1993, pp. 913-914.

[2] P.E. Kinahan and J.G. Rogers, Analytic three-dimensional image reconstruction using all detected events, IEEE Trans. Nucl. Sci. 361990 pp. 964-968.

[3] N.J. Pelc, A generalized filtered backprojection algorithm for 3D reconstruction, MIT, Boston, USA, Ph.D. dissertation, 1979.

[4] C. Comtat, M. Defrise, C. Morel and D. Townsend, The FaVoR algorithm for 3D PET data and its implementation using a network of transputers, Phys. Med. Biol. 38 (1993) pp. 929-944.

[5] M. Defrise, D.W. Townsend and R. Clack, FaVoR: a fast reconstruction algorithm for volume imaging in PET, in Conf. Records 1991 IEEE Medical Imag. Conf., Santa Fe, NM, 1992, pp. 1919-1923.

[6] D.W. Townsend, M. Wensveen, L.G. Byars, A. Geissbuhler, H.J. Tochon-Danguy, A. Christin, M. Defrise, D.L. Bailey, S. Grootoonk, A. Donath and R. Nutt, A rotating PET scanner using BGO block detectors: design, performance and applications, J. Nucl. Med. 34 (1993) pp. 1367-1376.

[7] D.W. Townsend, L.G. Byars, M. Defrise, A. Geissbuhler, R.P. Maguire, J. Missimer, R. Roddy, T. Brun, M.A. Mintun and R. Nutt. Design and performance of a rotating positron tomograph, RPT-2, in Conf. Records 1993 IEEE Medical Imag. Conf., San Francisco, CA, 1994, pp. 1058-1062.

[8] R.W. Dobinson, J.L. Pagès and J.C. Vermeulen, Transputers in particle physics experiments, Particle World, Vol. 2, No. 2 (1991) pp. 39-45.

J.L. Pagès, C. Comtat, C. Joseph, C. Morel, T.M. Tran, R.W. Dobinson, W. Huta, W. Lu, H. Wendler, C. Nicks and P. Schweizer, Parallel readout of the CERN RMH system using Transputers, Nuclear Instrum. Methods, A321 (1992) pp. 342-347.

[9] M.E. Casey and R. Nutt, A multicrystal two dimensional BGO detector system for positron emission tomography, IEEE Trans. Nucl. Sci. 33 (1986) pp. 460-463.

[10] T.J. Spinks, T. Jones, D.L. Bailey, D.W. Townsend, S. Grootoonk et al., Physical performance of a positron tomograph for brain imaging with retractable septa, Phys. Med. Biol, 37, (1992) pp. 1637-1655.

[11] Mark I. Williams, The corrections performed on the 3-D image data from a rotating PET scanner, by a new transputer-based system, which acquires and reconstructs this image data on-line, University of Liverpool, UK, MSc dissertation, 1994.

[12] E.J. Hoffman, T.M. Guerrero, G. Germano, W.M. Dighy and M. Dahlbom, PET system calibrations and corrections for quantitative and spatially accurate images, IEEE Trans. Nucl. Sci., 36 (1989) pp. 1108-1112.

[13] E.J. Hoffmann and M.E. Phelps, Positron emission tomography: principles and quantitation, in Positron emission tomography and autoradiography, principles and applications for the brain and heart, eds. M.E. Phelps, J.C. Maziotta and H.R. Schelbert (Raven Press, New York, 1986).

[14] F.F. de Jong, A.J.E. van Nieuwenborg and M.I. Williams, A parallel data handling system for a rotating positron tomograph using CHORUS and transputers, to be submitted.

[15] Inmos Ltd, 1988, Transputer reference manual, New York: Prentice-Hall.

[16] Inmos Ltd, 1991, IMS B016 VMEbus master card manual, Bristol, UK.

[17] Archipel S.A., 1992, VOLVOX TW8()() installation manual, Paris. 
[18] M. Rozier, V. Abrossimov, F. Armand, I. Boule, M. Gien, M. Guillemont, F. Herrmann, C. Kaiser, S. Langlois, P. Léonard, W. Neuhauser, Overview of the CHORUS distributed operating system, Chorus Systèmes, Paris, 1991.

[19] C. Comtat, M. Egger, A. Herrmann Scheurer, C. Morel, M. Defrise and D.W. Townsend, Use of the 3D FaVoR algorithm with large aperture PET scanners, in Conf. Records 1993 IEEE Medical Imag. Conf., San Francisco, CA, 1994, pp.16771681. 


\section{Figure Captions}

Fig. 1 Oblique line of response (LOR) making an angle $\pi / 2-\theta$ with the scanner axis. The angle $\theta$ is related to the ring index difference $i-j$. The LOR represented on this figure is parallel to the direction given by the aperture angle $\theta$ and the azimuthal angle $\phi$.

Fig. 2 (a) Diamond-shaped region acquired within each sinogram for a given angular position of the detector arrays. (b) As the detector arrays rotate, the diamond-shaped regions shift down the angular ordinate axis of the sinograms. (c) The actual radial extent of the useful field of view depends on the overlap hetween consecutive angular positions of the detector arrays. The figure illustrates two different acquisitions or scans carried out in 6 and 12 angular positions.

Fig. 3 Illustration of a 6-angular position scan. Once the detector arrays have rotated by $60^{\circ}$ (i.e. after the second angular position), a rectangular sinogram area-shown in whiteremains unaffected by subsequent coincidence detections and is therefore ready for image reconstruction. Sinogram areas shown in light grey represent rows that were completed at an earlier position.

Fig. 4 On-line parallel processing system for the rotating positron tomograph RPT-1. Coincidences are built from single events by commercial Siemens-CTI electronics and are stored in VME memory. The VME memory is read out by a B016 transputer-based VME module. Corrections are then applied in parallel by two T805 transputers, which also sort the data into sinograms. Sinogram data are sent to the buffer-transputers (VOLVOX-TW8(00) to await treatment by the reconstruction network.

Fig. 5 (a) Transputer-based reconstruction network. Oblique projections are processed on the left-hand side of the network and direct projections on the right-hand side. The number of transputers on each side is chosen so as to balance reconstruction times of oblique and direct projections. (b) The inherent difference of treatment between oblique and direct projections results in different image volume partitions used to parallelize reconstruction. With the oblique projections, each processor reconstructs the same strip of every image slice. With the direct projections, every processor reconstructs a number of complete transaxial slices.

Fig. 6 Timings and data volumes for a 12-angular position scan with an acquisition time of 12 minutes. The horizontal axis represents time, shown in minutes. Processings of oblique and direct projections are represented above and under the time axis, respectively. The evolution with time of the volume of sinogram data stored in the buffer, undergoing image reconstruction and already processed may be followed. The first set of data hecomes available for reconstruction 4 minutes after the start of the scan. This set of data is then corrected and assembled into sinogram rows before reconstruction can start. Transfer times of data from the buffer transputers to the reconstruction network are shown in dark grey. Reconstruction ends only 13.5 minutes after the end of the acquisition, although the computation time for the reconstruction is about 20 minutes. 


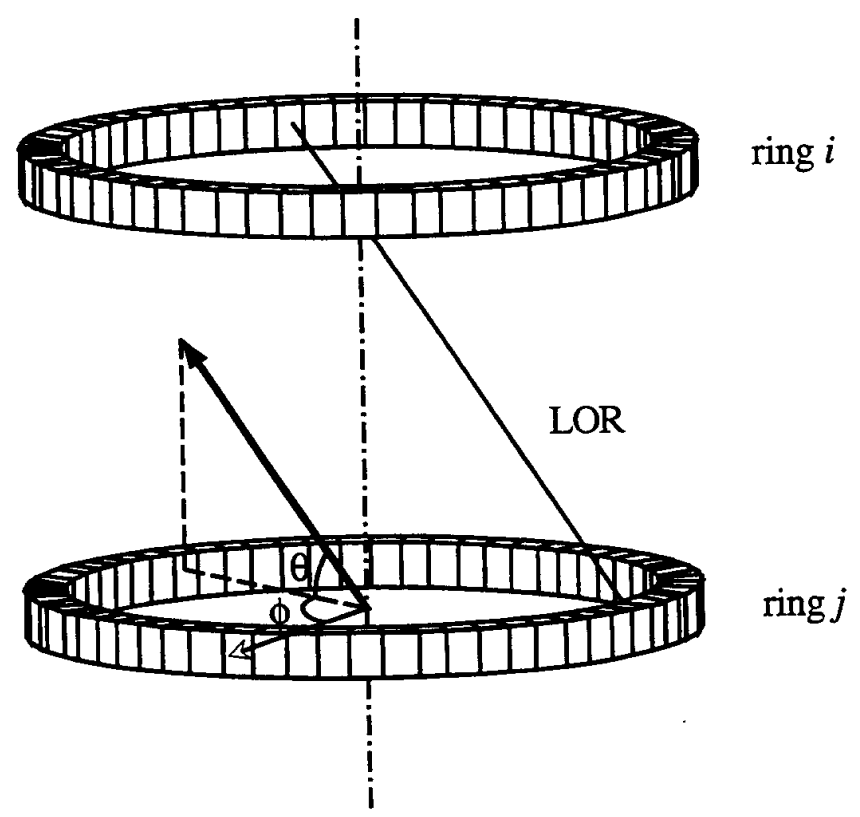

Fig. 1 


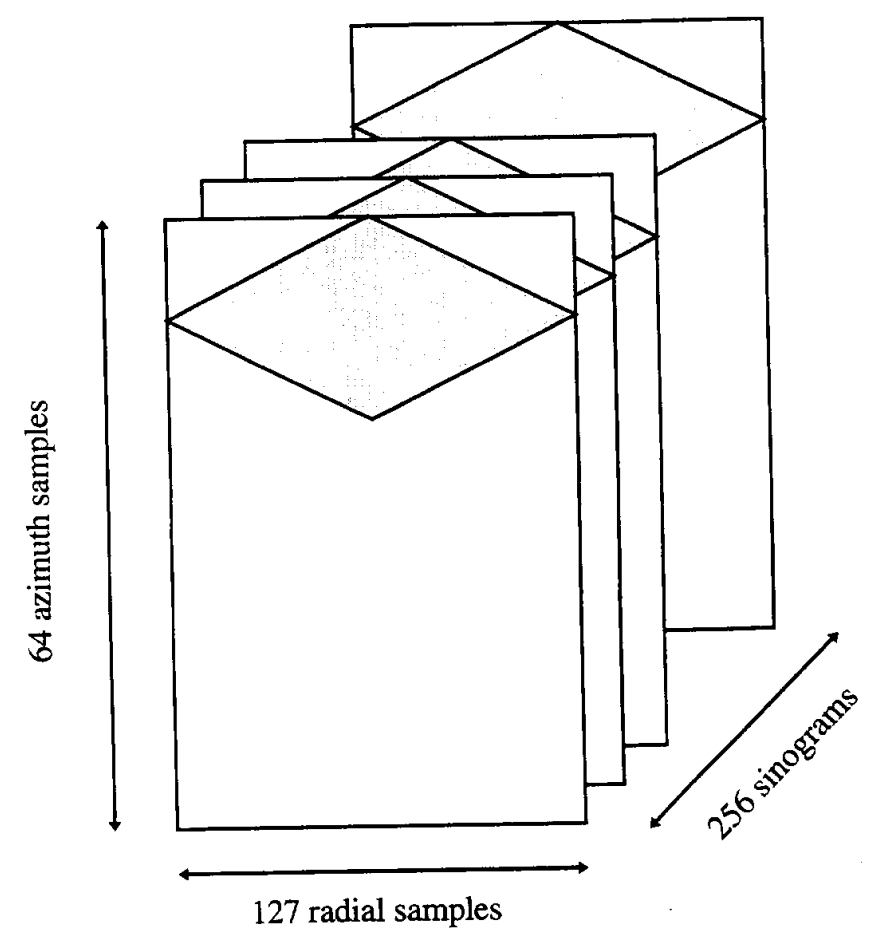

Fig. 2a
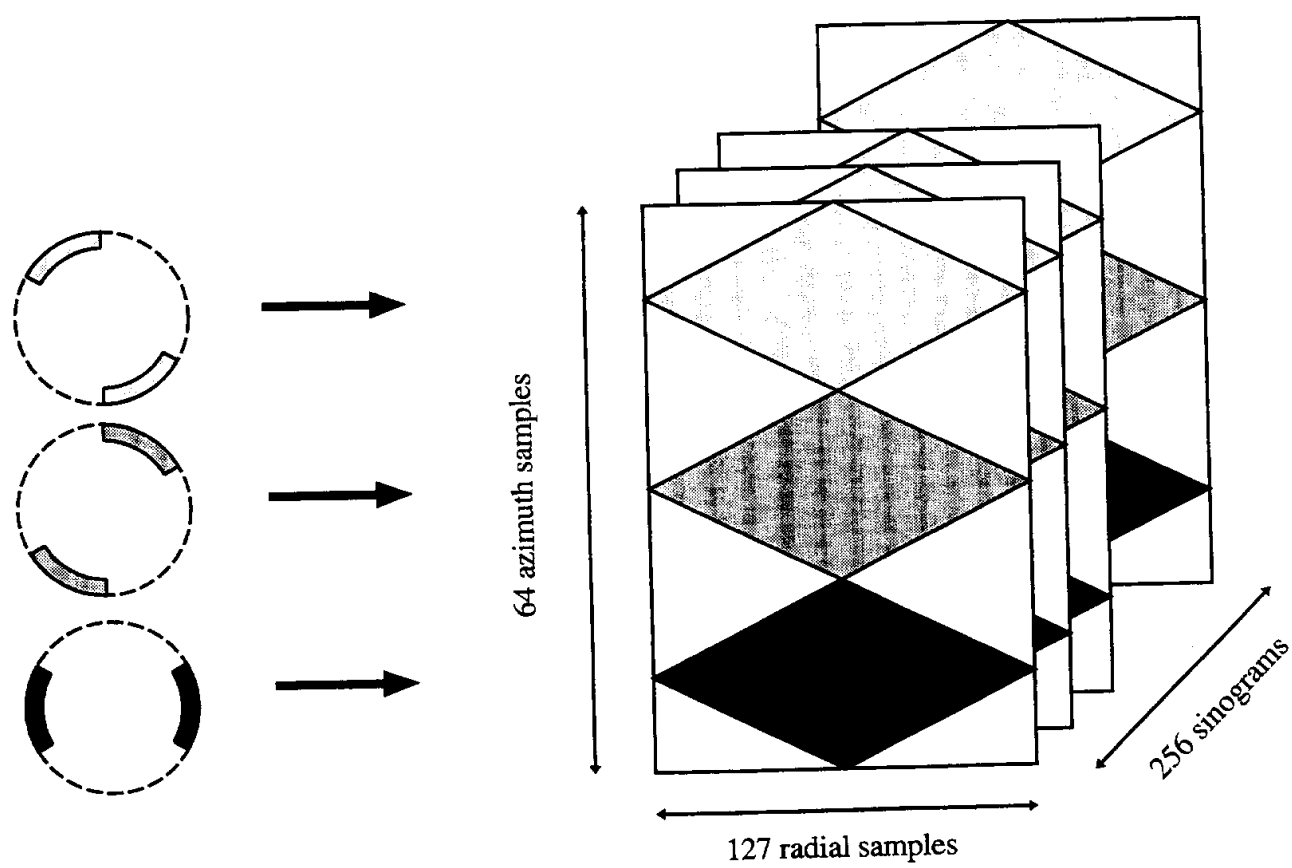

Fig. 2b 
6 angular position scan

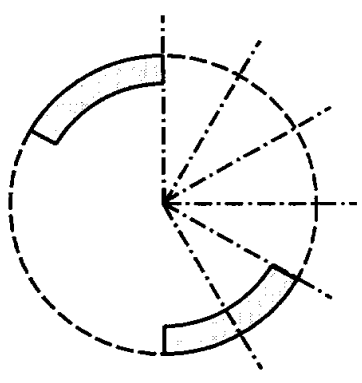

12 angular position scan

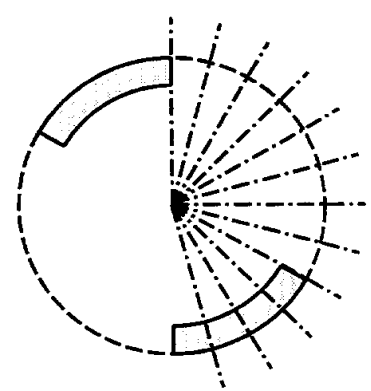

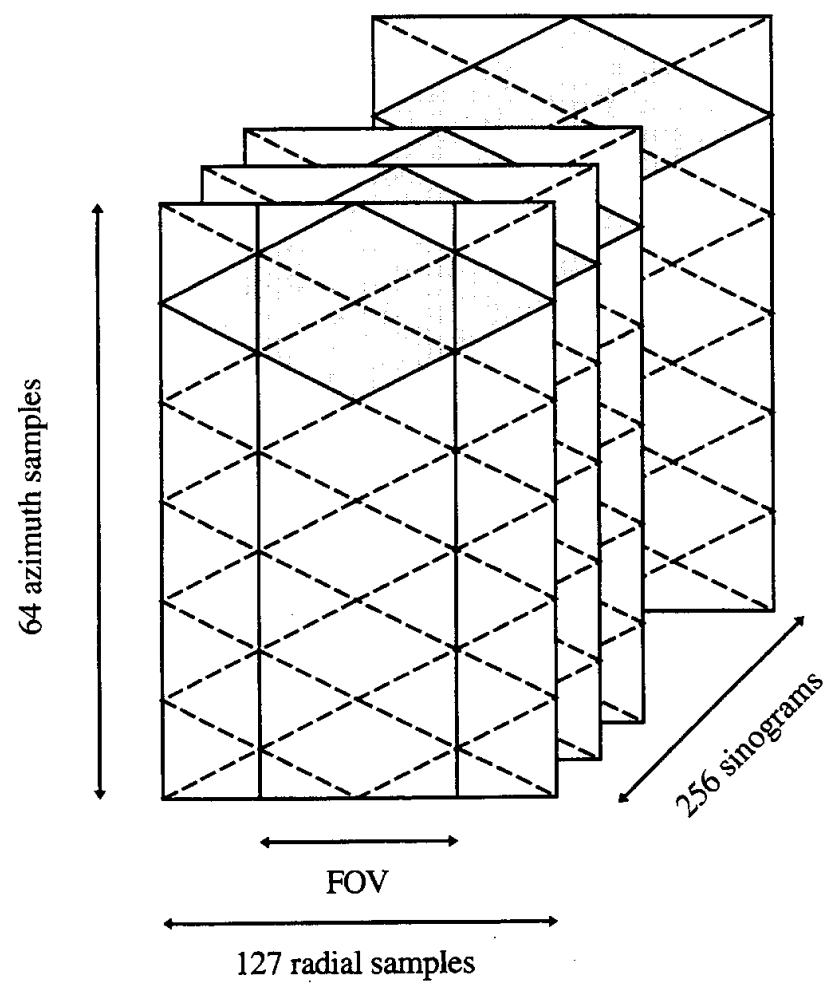

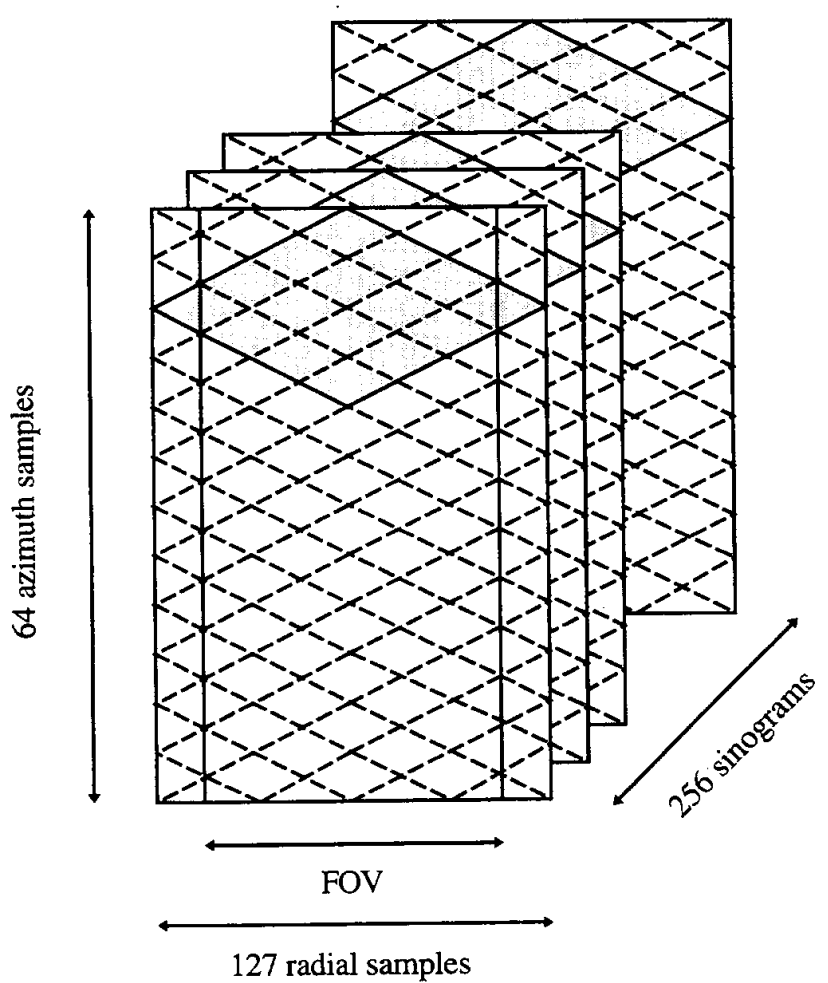

Fig. 2c 


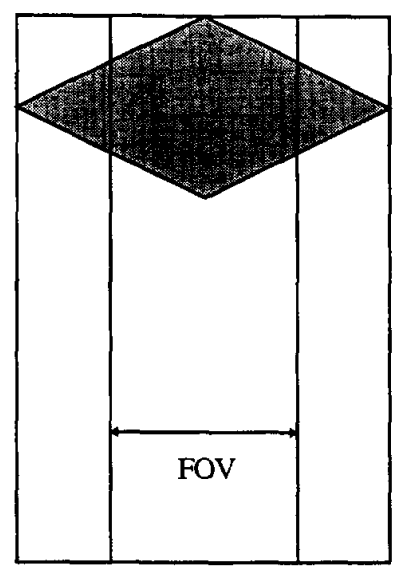

1st angular position

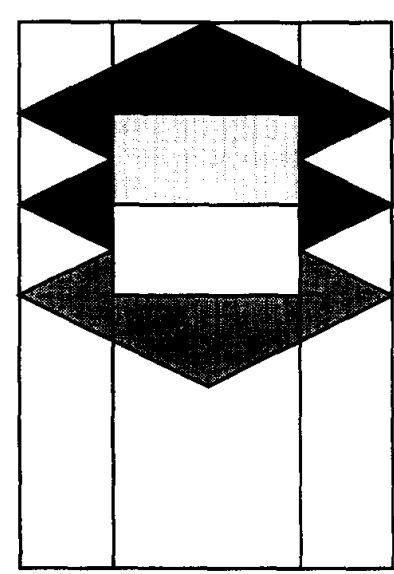

3rd angular position

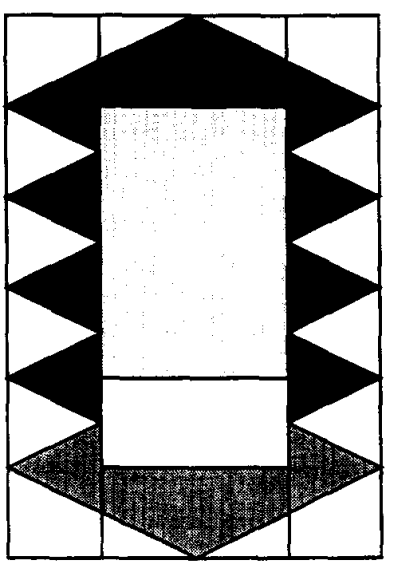

5th angular position

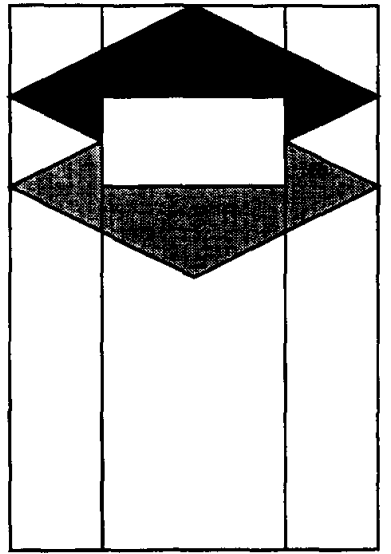

2nd angular position

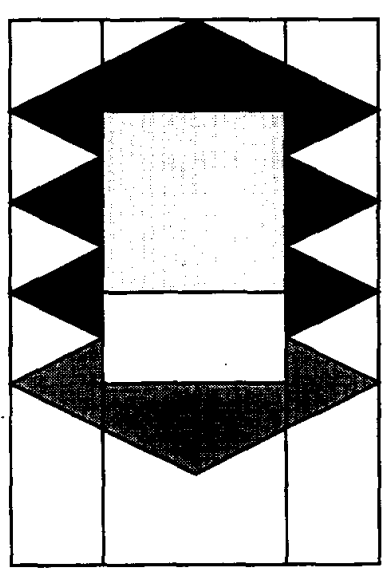

4th angular position

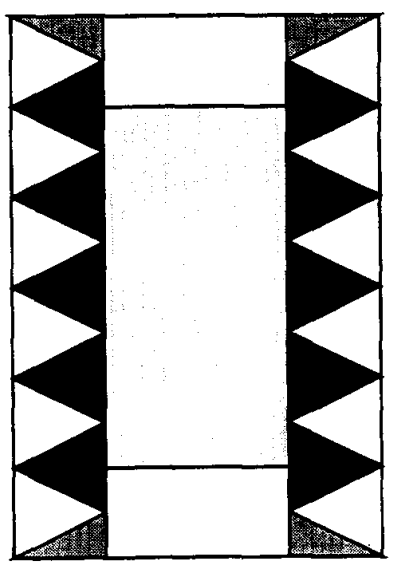

6th angular position

Fig. 3 


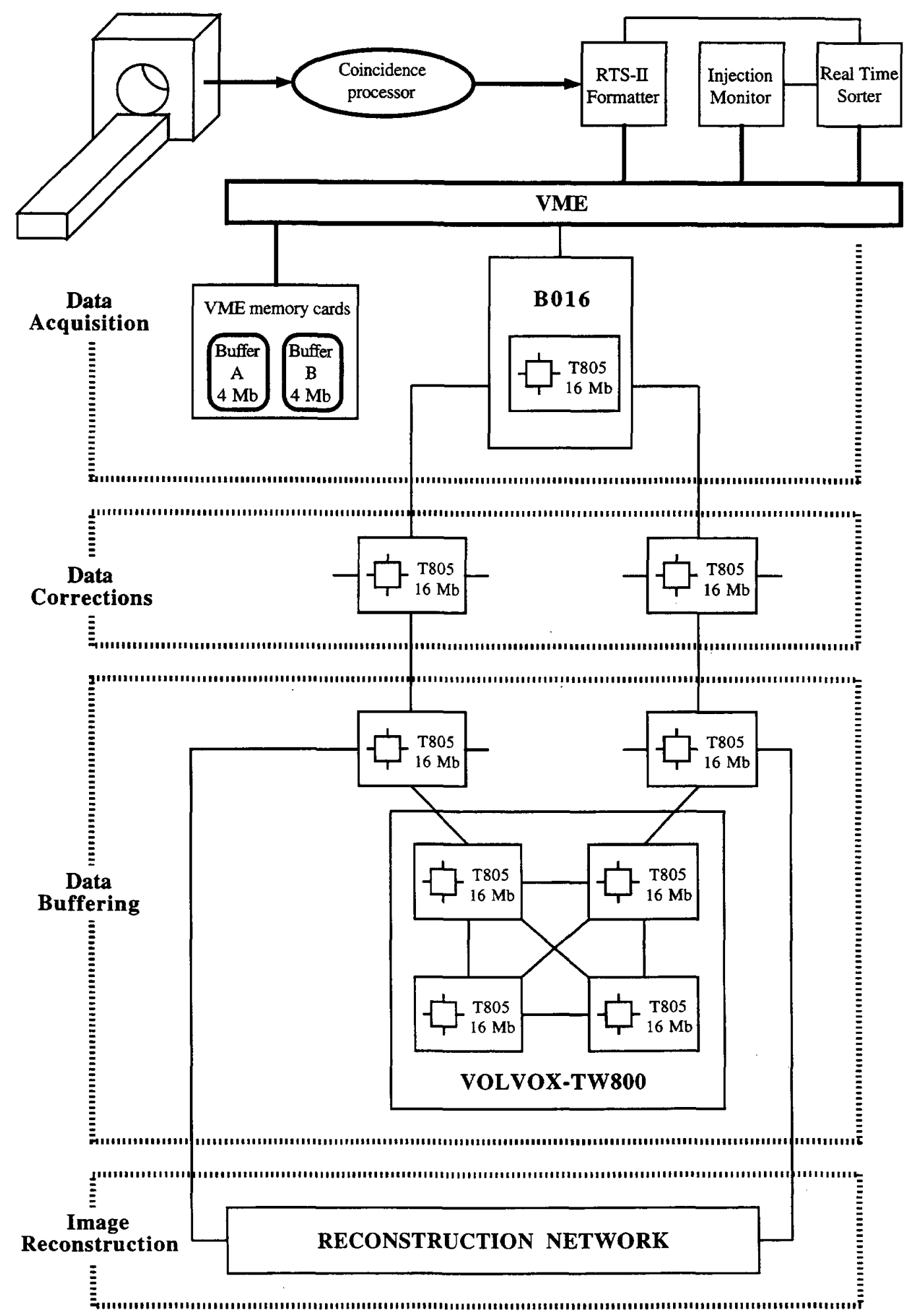

Fig. 4 


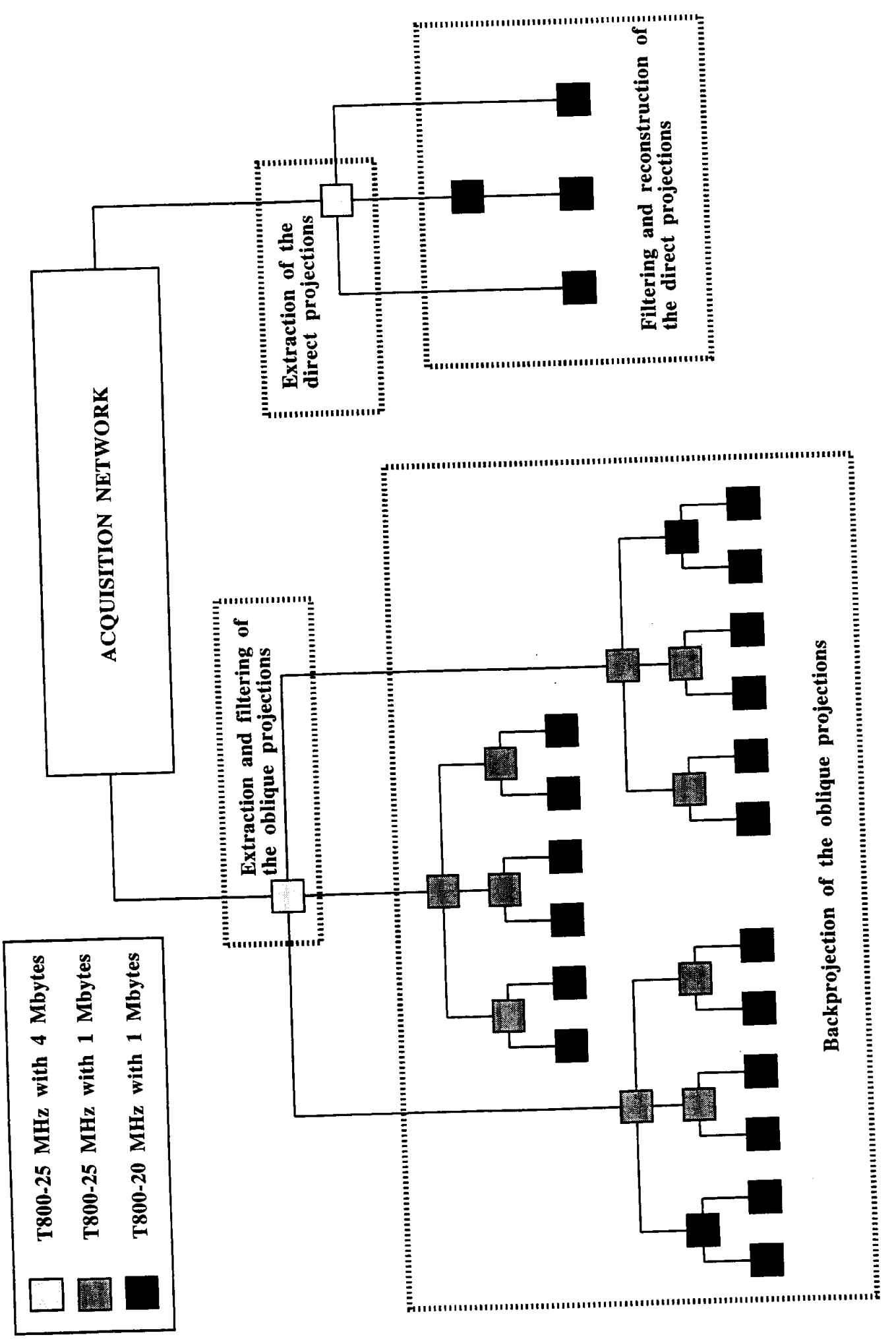

Fig. 5a 
Image volume
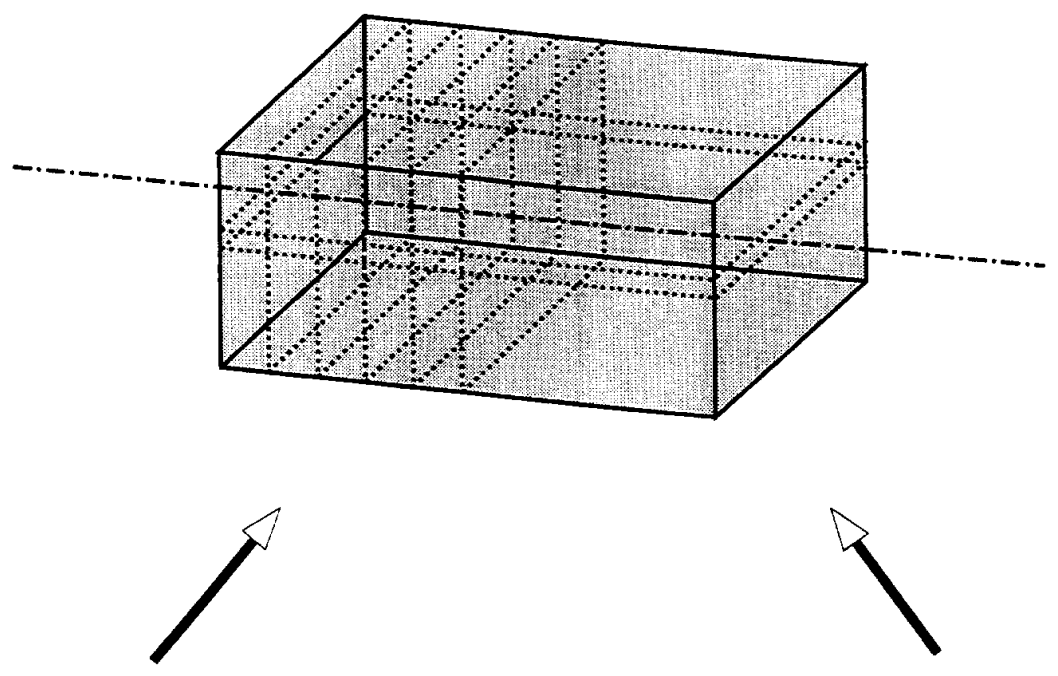

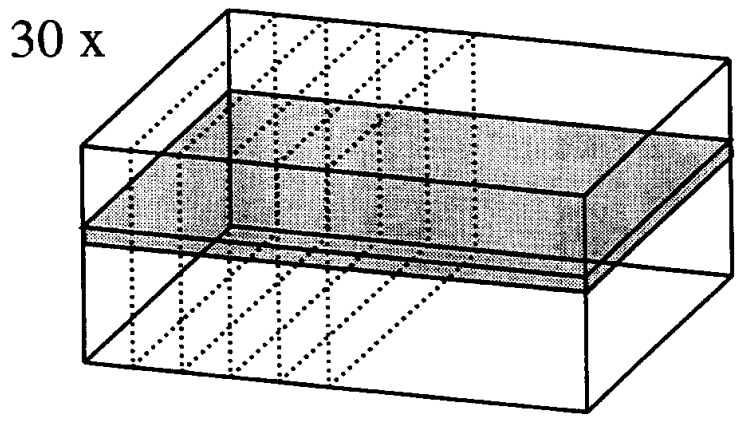

Processing of oblique projections

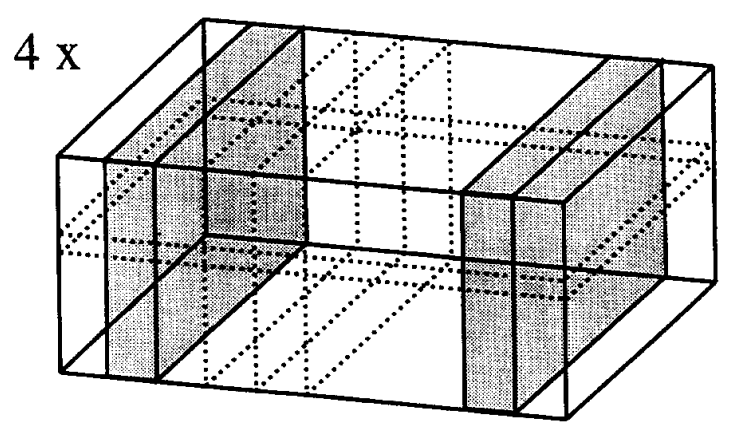

Processing of direct projections

Fig. 5b 


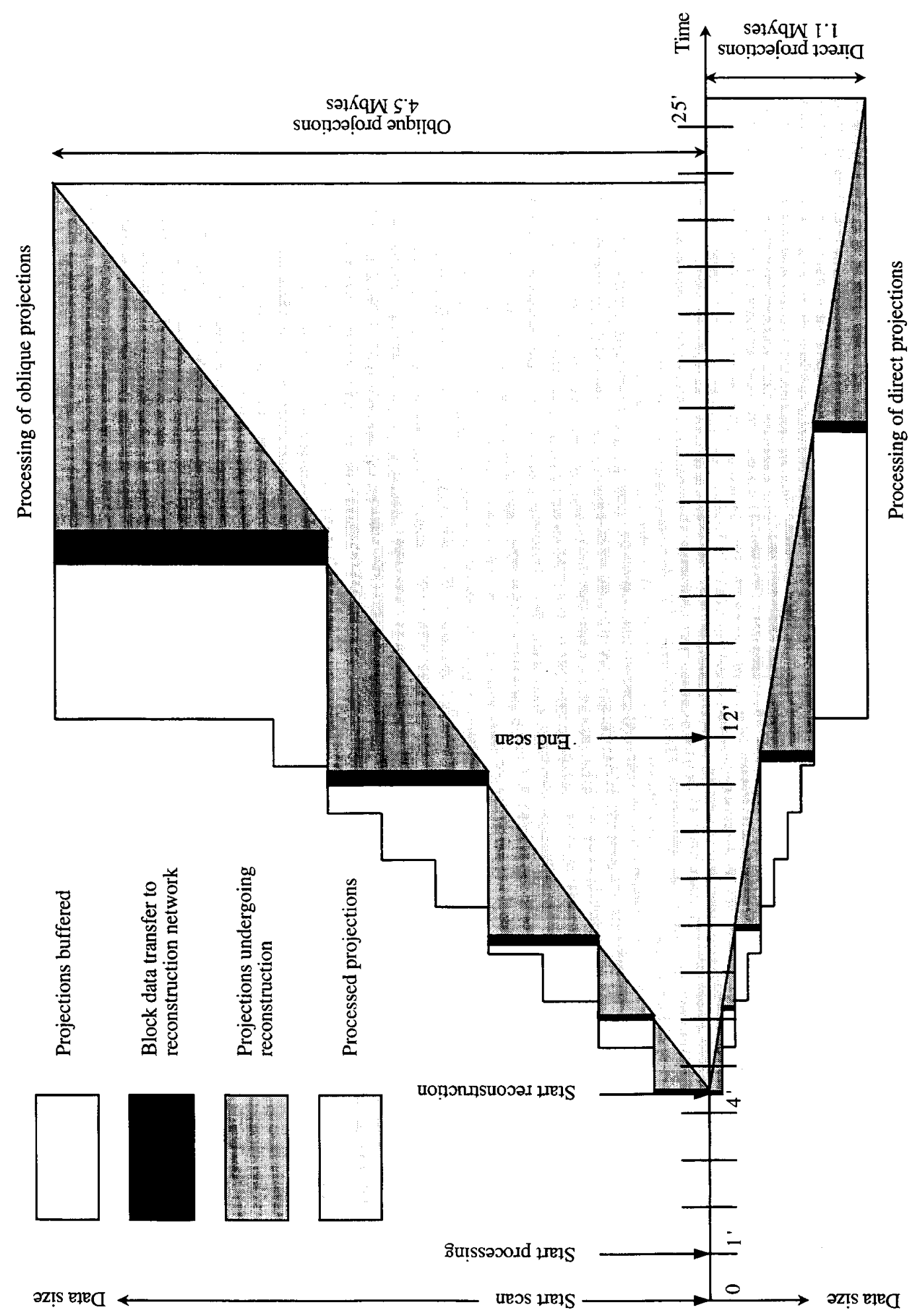

Fig. 6 
